\section{Chemistry \\ Far from simple kinetics}

\section{from J. Christopher Whitehead}

DESPITE the apparent simplicity of the exchange reaction $\mathrm{H}+\mathrm{H}_{2} \rightarrow \mathrm{H}_{2}+\mathrm{H}$, it has been a formidable task to check, experimentally, the theoretical predictions of how the energy of the reaction becomes distributed among the possible quantum states of the products and the directions in which the products recoil. Recent developments in laser technology have at least enabled two independent research groups to measure the energy of the very closely related reaction, $\mathrm{H}+\mathrm{D}_{2} \rightarrow \mathrm{HD}+\mathrm{D}$.

Because it is the simplest of all possible chemical reactions involving neutral species, $\mathrm{H}+\mathrm{H}_{2} \rightarrow \mathrm{H}_{2}+\mathrm{H}$ has played an important part in the development of the theory of chemical kinetics. The theoretical prediction of the outcome of the reaction proceeded in two stages. First, the energy of interaction of the three hydrogen atoms involved in the reaction was evaluated for all possible geometries. As a result of the considerable computational problems involved, it was not until 1956 that a truly $a b$ initio quantum mechanical calculation was completed (Boys, S.F. et al. Nature $178,1207)$ and not until 1978 that the calculations had been refined to the point where they are believed to be accurate for all configurations (Siegbahn, P. \& Liu, B. J. chem. Phys. 68, 2457).

The second stage of the theoretical studies used the calculated interaction energies in a quantum mechanical prediction of the paths that connect the reactants and the products and hence the distribution of the energy of the reaction among the possible quantum states of the products and the directions in which the products

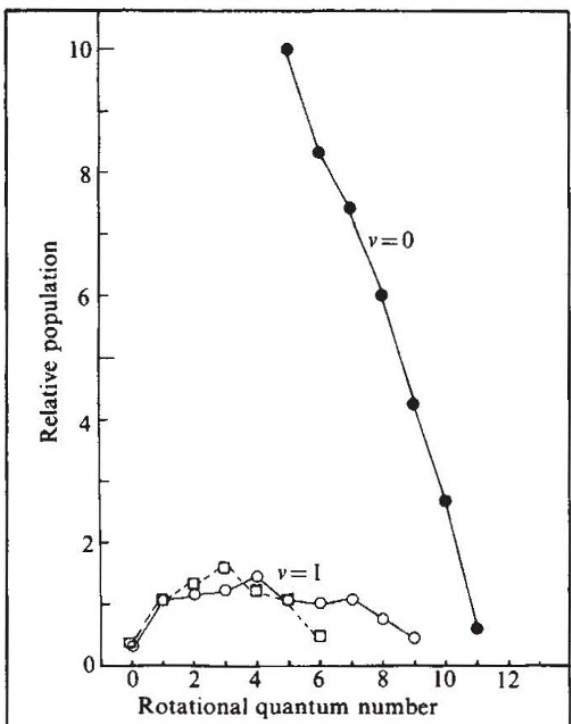

Relative populations of HD vibrational and rotational quantum states from the reaction $\mathrm{H}+\mathrm{D}_{2} \rightarrow \mathrm{HD}+\mathrm{D}$. Data from Zare's experiments are shown as squares, Valentini's as circles.

\section{Immunology}

from N.A. Mitchison will recoil (Schatz, G. \& Kupperman, A.J. J. chem. Phys. 65, 4668; 1976).

That these predictions have remained untested until now is in part because there is a barrier of $0.42 \mathrm{eV}$ to be overcome before reaction can take place and in part because it is difficult to generate a sufficient concentration of hydrogen atoms of high enough energy. Moreover, $\mathrm{H}_{2}$ is a difficult molecule to detect spectroscopically. To surmount those barriers, two US research groups have now used laser technology to produce fast $\mathrm{H}$ atoms in the presence of $\mathrm{D}_{2}$ to study the energy of the resulting HD product from the reaction $\mathrm{H}+\mathrm{D}_{2} \rightarrow \mathrm{HD}+\mathrm{D}$. Both groups generated the $\mathrm{H}$ atoms by photodissociating hydrogen iodide in the presence of deuterium, $D_{2}$, in the gas phase at a low pressure using the output of a highpower pulsed ultraviolet laser at $266 \mathrm{~nm}$. The production of HD was measured within 5-100 ns after the photolysis laser pulse to ensure that it had no time to undergo any further collisions.

The two groups employed different methods to detect the energy states of the HD product. One, led by R.N. Zare (Stanford University), used a technique in which the HD absorbs several photons from a tunable pulsed ultraviolet laser and becomes ionized; the results were reported at the XIIth International Conference on the Physics of Electronic and Atomic Collisions, in Berlin last summer. The other group, led by J.J. Valentini (Los Alamos National Laboratory), used a form of Raman spectroscopy (Gerrity, D.P. \& Valentini, J.J. J. chem. Phys. 79, 5202; 1983). Both methods give information on the relative populations of the rotational and vibrational energy levels of the HD product. As can be seen from the figure, there is close agreement between the results of the two groups. HD is produced principally in its lowest vibrational level $(v=0)$ with rotational distributions that peak at low quantum numbers. About 75 per cent of the energy available in the reaction is converted into kinetic energy of the reaction products as they fly apart.

The experiments represent a breakthrough in the study of chemical kinetics because they yield the first detailed information for a member of the $\mathrm{H}+\mathrm{H}_{2}$ family of reactions and demonstrate the feasibility of studying such fundamentally important reactions. This work will undoubtedly provide the stimulus for even more refined theoretical studies and advance our understanding of the basic principles of chemical kinetics.

J. Christopher Whitehead is in the Department of Chemistry, University of Manchester, Manchester M13 9PL.

\title{
Rational design of vaccines
}

THE late R.K. Gershon's major scientific legacy is to have demonstrated that the immune response, however simple it may look, is invariably the outcome of positive and negative regulatory influences mediated by helper and suppressor T cells. He showed that the key to understanding this balance is the experimental separation of the various helper and suppressor lymphocyte subsets, by means of alloantisera (such as anti-Lyt 2), monoclonal antibodies (such as T4 or T8), or lectins (for example, one from Vicia villosa reactive selectively with contrasuppressor cells), so that the regulatory properties of each cell type can be studied independently. Nowhere is this better illustrated than in the analysis of peripheral blood lymphocytes from patients with leprosy, reported by Mehra and his colleagues $^{1}$ in this issue of Nature (p.194).

Partitioning of these cells by means of T8 antibody reveals the presence, in lepromatous individuals, of a proliferativeresponder putatively helper $\mathrm{T}$-cell population which is prevented from acting by suppressor $T$ cells. This form of suppression offers a rational explanation for the continued progress of the disease in such cases. The determinant on Mycobacterium leprae with which the suppressor cells react is identified as a unique phenolic glycolipid. It can reasonably be assumed that the major infectious diseases afflicting the Third World, all of which are characterized by long-term interaction between host and parasite, involve a balance of this sort between regulatory lymphocytes. And if we knew how to design molecules able selectively to stimulate helper cells (or other positively acting regulatory cells such as contrasuppressors), we would be much further on the road to rational development of vaccines.

In a forthcoming paper, E. Sercarz and his group at the University of California, Los Angeles, bring their studies on lysozyme to a climax, with a definitive demonstration of a suppressor determinant at the $\mathrm{N}$ terminus of the protein ${ }^{2}$. Using a bacterial protease they cleaved off the three $\mathrm{N}$-terminal amino acids, leaving a molecule that was unable to induce suppressor $T$ cells under defined experimental conditions. Using slightly less direct methods, J. Levy and co-workers have 